\title{
Morphological Characterization of Greengram Germplasm using DUS Descriptors
}

\author{
S. Mounika, J. V. Ramana*, M. Lal Ahamed and D. Ratna Babu \\ Department of Molecular Biology and Biotechnology, Advanced Post Graduate Centre, \\ Acharya N G Ranga Agricultural University, Lam, Guntur, Andhra Pradesh, India \\ *Corresponding author
}

\section{A B S T R A C T}

\begin{tabular}{|l|}
\hline Ke y w o r d s \\
Greengram \\
Germplasm \\
DUS Descriptors \\
\hline Article Info \\
\hline $\begin{array}{l}\text { Accepted: } \\
\text { 15 August } 2020 \\
\text { Available Online: } \\
\text { 10 September } 2020\end{array}$ \\
\hline
\end{tabular}

\section{Introduction}

Green gram is a self-pollinating diploid grain legume $(2 \mathrm{n}=2 \mathrm{x}=22)$ crop with genome size of $543 \mathrm{Mb}$ (Kang et al., 2014) and the most important crop in India after chick pea and pigeon pea. It is a widely cultivated pulse crop, because of its adaptation to low water requirement, low soil fertility, short growth duration and can also be used in crop rotation practices. Other properties like easy digestibility and low proportions of flatulence factors also added to its value among the pulse crops. India is the primary producer of greengram and contributes to about $75 \%$ of the world production. In India, it contributes to about $14 \%$ of total pulse cultivation area and $7 \%$ of total production.

In India greengram is cultivated in an area of 4326.8 ha (957.21 ha in rabi and 3369.59 ha in kharif) with a production of 2165.36 tons (522.18 tons in rabi and 1643.18 tonns in kharif) and productivity of $1046 \mathrm{~kg} / \mathrm{ha}(546$ $\mathrm{kg} / \mathrm{ha}$ in rabi and $500 \mathrm{~kg} / \mathrm{ha}$ in kharif). In Andhra Pradesh, green gram productivity is $990 \mathrm{~kg} / \mathrm{ha}$ ( rabi is $490 \mathrm{~kg} / \mathrm{ha}$ and $500 \mathrm{~kg} / \mathrm{ha}$ in kharif) (Indiastat, 2016-17).

Genetic diversity is the base for crop genetic improvement and important for the conservation, evaluation and utilization of 
crop germplasm (Rao and Hodgkin, 2002; Wang et al., 2015 and Anumalla et al., 2015). Mung bean gene pool can be best utilized for development of promising or superior varieties by exhaustive characterization of the various germplasm holdings and collections that constitute the gene pools to identify the useful genetic diversity. The crosses between the parents with maximum genetic divergence are generally the most responsive for genetic improvement (Arunachalam, 1981). However, it is necessary to screen and characterize the germplasm for the nature and extent of genetic diversity to know the traits present in the selected parental genotypes.

Characterization and cataloguing of germplasm have been traditionally carried out by using morpho-agronomic traits. BioVersity International and PPV \& FRA (Protection of Plant Varieties and Farmers' Rights Authority) have come up with a set of DUS (Distinctiveness, Uniformity and Stability) descriptors for characterization of the lines for their registration and protection. Thus, in the present study, fifty genotypes collected from different parts of the country were characterized using PPV \& FRA descriptors to know the extent variability present in these genotypes.

\section{Materials and Methods}

The experimental material consisted of 48 mungbean genotypes collected from different parts of the country was evaluated at Advanced Post Graduate Centre, Lam, Guntur, Andhra Pradesh, India, to get the clear idea of variability present for different traits. The genotypes were planted in one row of $2 \mathrm{~m}$ length in three replications in a randomized block design. The plant to plant distance was $10 \mathrm{~cm}$ and row to row distance was $30 \mathrm{~cm}$. Standard agronomic practices were followed to raise a good crop and need based plant protection measures were adopted for managing good crop stand. The observations on morphological characters were recorded during different growth stages of the plant. The anthocyanin pigmentation at cotyledonary stages was observed at unfolded stages (5days after sowing). The characters like growth habit, plant habit, stem colour, leaf colour, vein colour, leaf size, petiole colour and stem pubescence were observed at $50 \%$ flowering stage. The characters like plant height and premature pod colour were observed when pods were fully developed while, pod colour at maturity, pod curvature, pod position, mature pod length were observed at maturity stage. Seed characters like seed colour, seed size, seed coat luster, seed shape were observed after harvest. All morphological observations were conducted as per the DUS testing guidelines issued by Protection of Plant Varieties \& Farmers Rights Authority (2007).

\section{Results and Discussion}

Morphological characteristics provide the basic information about the magnitude of genetic variability. 26 morphological characters were recorded in 48 greengram genotypes and variability was observed in 22 characters indicating the usefulness of these traits in differentiating the genotypes (Table 1 and 2). The trait, anthocyanin colouration, was recorded at seedling stage and was noticed in the genotypes, TM 96-2, GGG-1, IPM 2-14, LGG-617, WGG-42, LGG-450, LGG-625, LGG-605, OBGG-56, LGG-595, VBN-2, TARM-1, LGG-634, LGG-636, RM 16-9, OBGG-58, PM-5, Pusa Vishal. Pusa Ratna, IPM-288,IPM 02-3, IPM 02-14, MH 2-15,MH 96-1, MH-318, ML 1464, HUM-1, EC-398885, EC-398891, EC-520024, EC520026, EC-520029, China Mung, Butan LM -1, Prakash Nepal and VGG 15-30. Anthocyanin colouration was absent in 13 genotypes. 
Table.1 DUS descriptors of 48 Mungbean genotypes






\begin{tabular}{|c|c|c|c|c|c|c|c|c|c|c|c|c|c|c|c|c|c|c|c|c|c|c|c|c|c|c|}
\hline & 520024 & & & & & & & & & & & & & & & & & & & & & & & & & \\
\hline 40 & $\begin{array}{c}\text { EC - } \\
520026\end{array}$ & 2 & 3 & 5 & 1 & 3 & 9 & 1 & 2 & 1 & 2 & 1 & 3 & 1 & 3 & 3 & 1 & 9 & 1 & 3 & 3 & 3 & 2 & 1 & 3 & 5 \\
\hline 41 & $\begin{array}{c}\text { EC - } \\
520029\end{array}$ & 2 & 3 & 3 & 1 & 1 & 9 & 1 & 2 & 1 & 2 & 1 & 3 & 1 & 5 & 3 & 1 & 9 & 1 & 3 & 3 & 3 & 2 & 1 & 3 & 5 \\
\hline 42 & $\begin{array}{l}\text { China } \\
\text { Mung }\end{array}$ & 2 & 3 & 5 & 1 & 1 & 9 & 1 & 2 & 1 & 2 & 1 & 1 & 1 & 3 & 3 & 1 & 9 & 1 & 3 & 3 & 3 & 2 & 1 & 3 & 5 \\
\hline 43 & $\begin{array}{c}\text { Butan LM } \\
-1\end{array}$ & 2 & 3 & 3 & 1 & 1 & 9 & 1 & 2 & 1 & 2 & 3 & 1 & 1 & 5 & 3 & 2 & 9 & 1 & 3 & 3 & 3 & 2 & 1 & 3 & 5 \\
\hline 44 & $\begin{array}{c}\text { Prakash } \\
\text { Nepal }\end{array}$ & 2 & 3 & 3 & 1 & 1 & 9 & 1 & 2 & 1 & 2 & 1 & 3 & 1 & 3 & 3 & 1 & 9 & 1 & 3 & 3 & 3 & 2 & 1 & 3 & 3 \\
\hline 45 & LGG - 460 & 1 & 3 & 5 & 1 & 1 & 9 & 1 & 2 & 1 & 2 & 1 & 3 & 1 & 3 & 3 & 1 & 9 & 1 & 3 & 3 & 3 & 2 & 1 & 1 & 3 \\
\hline 46 & LGG - 644 & 1 & 3 & 5 & 1 & 1 & 9 & 1 & 2 & 1 & 2 & 1 & 3 & 2 & 5 & 3 & 1 & 9 & 1 & 3 & 7 & 3 & 2 & 1 & 3 & 5 \\
\hline 47 & LGG -407 & 1 & 3 & 3 & 1 & 1 & 9 & 1 & 2 & 1 & 2 & 1 & 3 & 2 & 5 & 3 & 1 & 9 & 1 & 3 & 7 & 3 & 2 & 2 & 1 & 5 \\
\hline \multirow[t]{3}{*}{48} & $\begin{array}{c}\text { VGG } 15 \text { - } \\
30\end{array}$ & 2 & 3 & 5 & 1 & 3 & 9 & 9 & 4 & 1 & 2 & 1 & 3 & 2 & 5 & 3 & 1 & 9 & 1 & 3 & 3 & 3 & 2 & 1 & 1 & 3 \\
\hline & & Absent-1 & Early-3 & Erect-3 & $\begin{array}{c}\text { Determinate- } \\
1\end{array}$ & Green-1 & Absent-1 & $\begin{array}{c}\text { Absent- } \\
1\end{array}$ & - deltoid- 1 & $\begin{array}{c}\text { Green- } \\
1\end{array}$ & $\begin{array}{c}\text { Brown- } \\
1\end{array}$ & -Straight-1 & 1 Green-1 & Green-1 & Small-3 & $\begin{array}{c}\text { Yellow- } \\
3\end{array}$ & - Green-1 A & $\begin{array}{c}\text { Absent- } \\
1\end{array}$ & $\begin{array}{c}\text { Above } \\
\text { canopy-1 }\end{array}$ & Short-3 & Early-3 & small-3 & $\begin{array}{c}\text { Yellow- } \\
1\end{array}$ & $\begin{array}{c}\text { Shiny- } \\
1\end{array}$ & - Oval-1 & 1 Small-3 \\
\hline & & Present -2 & $\begin{array}{l}2 \text { Medium- } \\
5 \text { Late-7 }\end{array}$ & $\begin{array}{c}\text { Semi } \\
\text { erect-5 } \\
\text { Spreading- } \\
7\end{array}$ & $\begin{array}{c}\text { In } \\
\text { determinate- } \\
3\end{array}$ & $\begin{array}{c}\text { Green } \\
\text { with } \\
\text { purple-2 } \\
\text { purple-3 }\end{array}$ & Present-9 & $\begin{array}{l}\text { Present- } \\
9\end{array}$ & $\begin{array}{c}\text { Ovate-2 } \\
\text { Lanceolate- } \\
3 \text { Cuneate- } \\
4\end{array}$ & $\begin{array}{c}\text { Dark } \\
\text {-green-2 }\end{array}$ & $\begin{array}{l}\text { Black- } \\
2\end{array}$ & Curved-3 & $\begin{array}{r}3 \text { Greenish } \\
\text { purple-2 } \\
\text { Purple-3 }\end{array}$ & $\begin{array}{l}\text { hGreenishN } \\
2 \text { with } \\
3 \text { purple - } \\
2 \text { Purple } \\
-3\end{array}$ & $\begin{array}{c}\text { hedium- } \\
5 \\
\text { Large-7 }\end{array}$ & $\begin{array}{c}\text { Light } \\
\text { yellow } \\
-5\end{array}$ & $\begin{array}{c}\text { Green } \\
\text { with } \\
\text { pigmented } \\
-2\end{array}$ & $\begin{array}{l}\text { Present Ir } \\
-9\end{array}$ & $\begin{array}{c}\text { Intermediate- } \\
2 \\
\text { Not visible- } \\
3\end{array}$ & $\begin{array}{c}\text {-medium- } \\
5 \\
\text { Large-7 }\end{array}$ & $\begin{array}{l}- \text { medium-7 } \\
5 \text { large-7 }\end{array}$ & $\begin{array}{c}7 \text { medium- } \\
5 \\
\text { large- }\end{array}$ & $\begin{array}{r}\text { a-Green-2 } \\
\text { mottled- } \\
3 \\
\text { Black-4 }\end{array}$ & Dull-2 & $\begin{array}{c}\text { Drum- } \\
3\end{array}$ & $\begin{array}{l}\text { Medium- } \\
5 \text { Large- } \\
7\end{array}$ \\
\hline
\end{tabular}

Table.2 Grouping of 48 mungbean genotypes based on DUS descriptors

\begin{tabular}{|c|c|l|}
\hline $\begin{array}{c}\text { Morphological } \\
\text { characters }\end{array}$ & Character state & \multicolumn{1}{c|}{ Genotypes } \\
\hline $\begin{array}{c}\text { Hypocotyl } \\
\begin{array}{c}\text { Anthocyanin } \\
\text { colouration }\end{array}\end{array}$ & Absent & $\begin{array}{l}\text { LGG-600, LGG-610, LGG-604, LGG-509, PM-110, MGG-385, LGG6-30, LGG-607, LGG-603, LGG-578, LGG-460, } \\
\text { LGG6-44, LGG-407 }\end{array}$ \\
\cline { 2 - 3 } & Present & $\begin{array}{l}\text { TM 96-2, GGG-1, IPM 2-14, LGG-617, WGG-42, LGG-450, LGG-625, LGG-605, OBGG-56, LGG-595, VBN-2, } \\
\text { TARM-1, LGG-634, LGG-636, RM 16-9, OBGG-58, PM-5, PUSA VISHAL, PUSA RATNA, IPM-288, IPM 02-3, MH } \\
\text { 2-15, MH 96-1, MH-318, ML-1464, HUM-1, EC-39885, EC-398891, EC-520024, EC-520026, EC-520029, CHINA } \\
\text { MUNG, BUTAN LM-1, PRAKASH NEPAL, VGG 15-30 }\end{array}$ \\
\hline $\begin{array}{c}\text { Plant growth } \\
\text { habit }\end{array}$ & Erect & $\begin{array}{l}\text { LGG-600, TM 96-2, LGG-610, LGG-604, GGG-1, IPM 2-14, LGG-617, WGG-42, PM-110, LGG-605, MGG-385, LGG- } \\
\text { 595, LGG-630, VBN-2, OBGG-58, PM-5, PUSA VISHAL, MH 96-1, EC-520024, EC-520029, BUTAN LM-1, } \\
\text { PRAKASH NEPAL, LGG-407 }\end{array}$ \\
\cline { 2 - 3 } & Semi- erect & $\begin{array}{l}\text { LGG-450, LGG-509, LGG-625, OBGG-56, TARM-1, LGG-634, LGG-636, LGG-607, RM 16-9, LGG-603,LGG-578, } \\
\text { PUSA RATNA, IPM-288, IPM 02-3, MH- 215, MH-318, ML-1464, HUM-1, EC-39885, EC-398891, EC-520026 CHINA } \\
\text { MUNG, LGG-460, LGG-644, VGG 15-30 }\end{array}$ \\
\hline
\end{tabular}




\begin{tabular}{|c|c|c|}
\hline Plant habit & Determinate & All genotypes \\
\hline \multirow[t]{3}{*}{ Petiole colour } & Green & $\begin{array}{l}\text { LGG-600, TM 96-2, LGG-604, IPM 2-14, LGG-617, WGG-42, PM-110, MGG-385, LGG-595, LGG-634, LGG-636, } \\
\text { LGG-607, RM 16-9, LGG-578, PM-5, IPM-288, MH 2-15, HUM-1, EC-39885, EC-398891, EC-520026, EC-520029, } \\
\text { CHINA MUNG, BUTAN LM-1, PRAKASH NEPAL, LGG-460 }\end{array}$ \\
\hline & $\begin{array}{l}\text { Green with purple } \\
\text { splashes }\end{array}$ & $\begin{array}{l}\text { LGG-610, GGG-1, LGG-450, LGG-509, LGG-625, OBGG-56, LGG-630, VBN-2, TARM-1, LGG-603, OBGG-58, } \\
\text { PUSA VISHAL, PUSA RATNA, IPM 02-3, MH 96-1, MH-318, ML-1464, EC-520024, LGG-644, LGG-407, VGG 155- } \\
30\end{array}$ \\
\hline & Purple & LGG-605 \\
\hline \multirow[t]{2}{*}{ Plant height } & Short & $\begin{array}{l}\text { LGG-600, IPM 2-14, LGG-617, WGG-42, OBGG-56, LGG-595,, VBN-2, TARM-1, LGG-634, LGG-636, LGG-607, } \\
\text { PM-5, PUSA VISHAL, PUSA RATNA, IPM-288, IPM 02-3, MH-215, MH 96-1, MH-318, ML-1464, HUM-1, EC- } \\
\text { 39885, EC-398891, EC-520024, EC-520026 EC-520029, CHINA MUNG, BUTAN LM-1, PRAKASH NEPAL, LGG- } \\
\text { 460, LGG-644, LGG-407, VGG 15-30 }\end{array}$ \\
\hline & Medium & $\begin{array}{l}\text { TM 96-2, LGG-610, LGG-604, GGG-1, LGG-450, LGG-509, LGG-625, PM-110, LGG-605, MGG-385, LGG-630, RM } \\
\text { 16-9, LGG-603, OBGG-58, LGG-578, }\end{array}$ \\
\hline \multirow[t]{3}{*}{ Stem colour } & Green & $\begin{array}{l}\text { GGG-1, IPM 2-14, LGG-617, WGG42, LGG-450, LGG-509, LGG-625, PM-110, LGG-605, OBGG-56, MGG-385, } \\
\text { LGG-595, LGG-630, VBN-2, TARM-1, LGG-634, LGG-636, LGG-607, RM 16-9, LGG-603, OBGG-58, LGG-578, PM- } \\
\text { 5, PUSA VISHAL, PUSA RATNA, IPM 02-3, MH-215, MH-318, ML-1464, HUM-1, EC-39885, EC-398891, EC- } \\
\text { 520024, EC-520029, CHINA MUNG, BUTAN LM-1, PRAKASH NEPAL, LGG-460, LGG-644, LGG-407 }\end{array}$ \\
\hline & $\begin{array}{l}\text { Green with purple } \\
\text { splashes }\end{array}$ & IPM-288 \\
\hline & Purple & LGG-600, TM 96-2, LGG-610, LGG-604, MH 96-1, EC-520026, VGG 15-30 \\
\hline Stem pubescence & Present & All genotypes \\
\hline \multirow[t]{2}{*}{$\begin{array}{l}\text { Leaflet lobes } \\
\text { (terminal) }\end{array}$} & Absent & $\begin{array}{l}\text { TM 96-2, LGG-610, LGG-604, GGG-1, IPM 2-14, LGG-617, WGG-42, LGG-450, LGG-590, LGG-625, PM-110, LGG- } \\
\text { 605, OBGG-56, MGG-385, LGG-595, LGG-630, TARM-1, LGG-634, LGG-636, LGG-607, LGG-603, OBBGG-58, } \\
\text { LGG-578, PM-5, PUSA VISHAL, PUSA RATNA, IPM-288, IPM 02-3, MH-215, MH 96-1, MH-318, ML-1464, HUM- } \\
\text { 1, EC-39885, EC-398891, EC-520024, EC-520026, EC-520029, CHINA MUNG, BUTAN LM-1, PRAKASH NEPAL, } \\
\text { LGG-460, LGG-644, LGG-407 }\end{array}$ \\
\hline & Present & LGG-600, VBN-2, RM 16-9, VGG 15-30 \\
\hline $\begin{array}{l}\text { Leaf shape } \\
\text { (terminal) }\end{array}$ & Ovate & $\begin{array}{l}\text { TM 96-2, LGG-610, LGG-604, GGG-1, IPM 2-14, LGG-617, WGG-42, LGG-450, LGG-509, LGG-625, PM-110, LGG- } \\
\text { 595, LGG-630, TARM-1, LGG-634, LGG-636, LGG-607, LGG-603, OBBGG-58, LGG-578, PM-5, PUSA VISHAL, } \\
\text { PUSA RATNA, IPM-288, IPM 02-3, MH-215, MH 96-1, MH-318, ML-1464, HUM-1, EC-39885, EC-398891, EC- } \\
\text { 520024, EC-520026, EC-520029, CHINA MUNG, BUTAN LM-1, PRAKASH NEPAL, LGG-460, LGG-644, LGG-407 }\end{array}$ \\
\hline
\end{tabular}




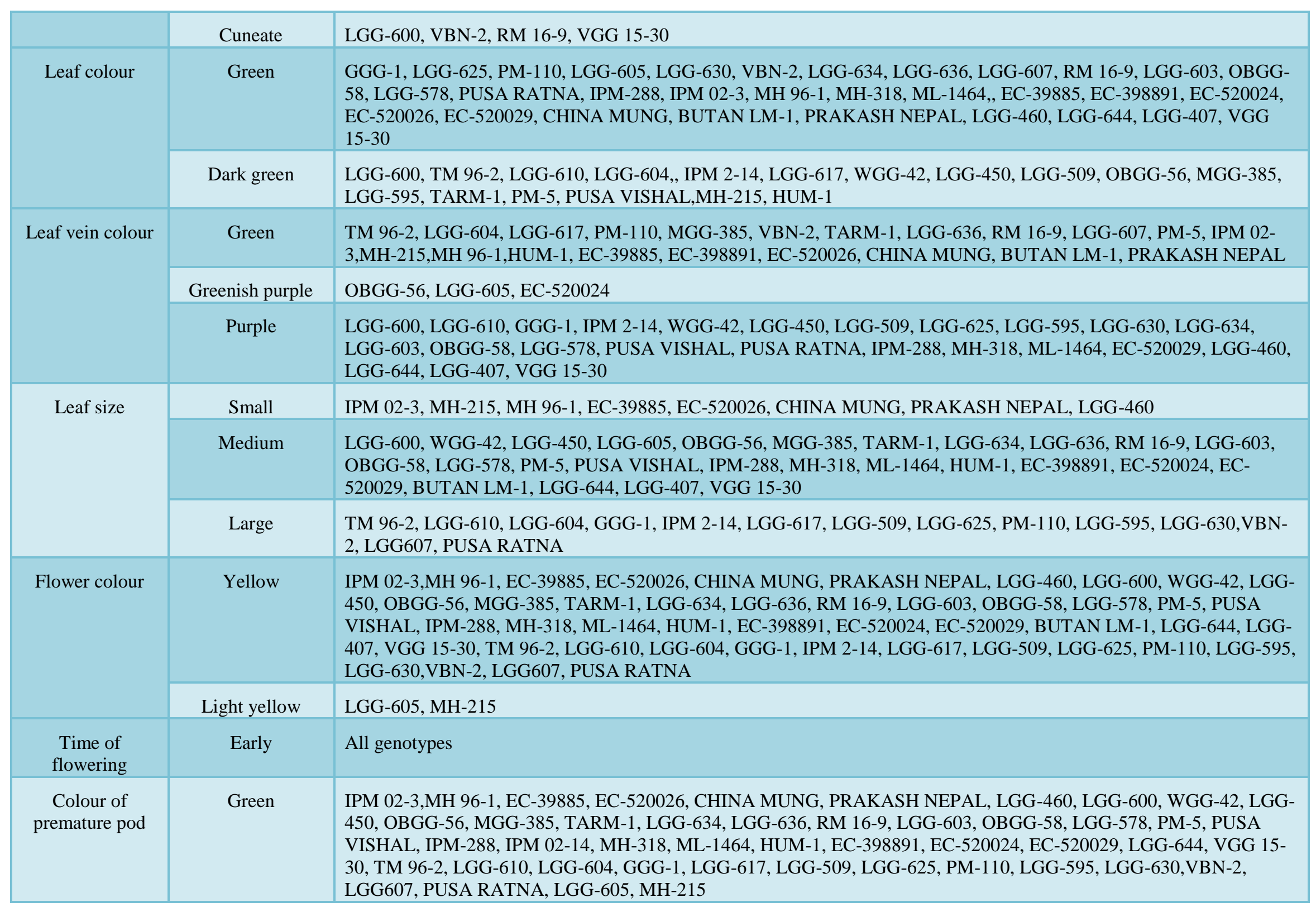




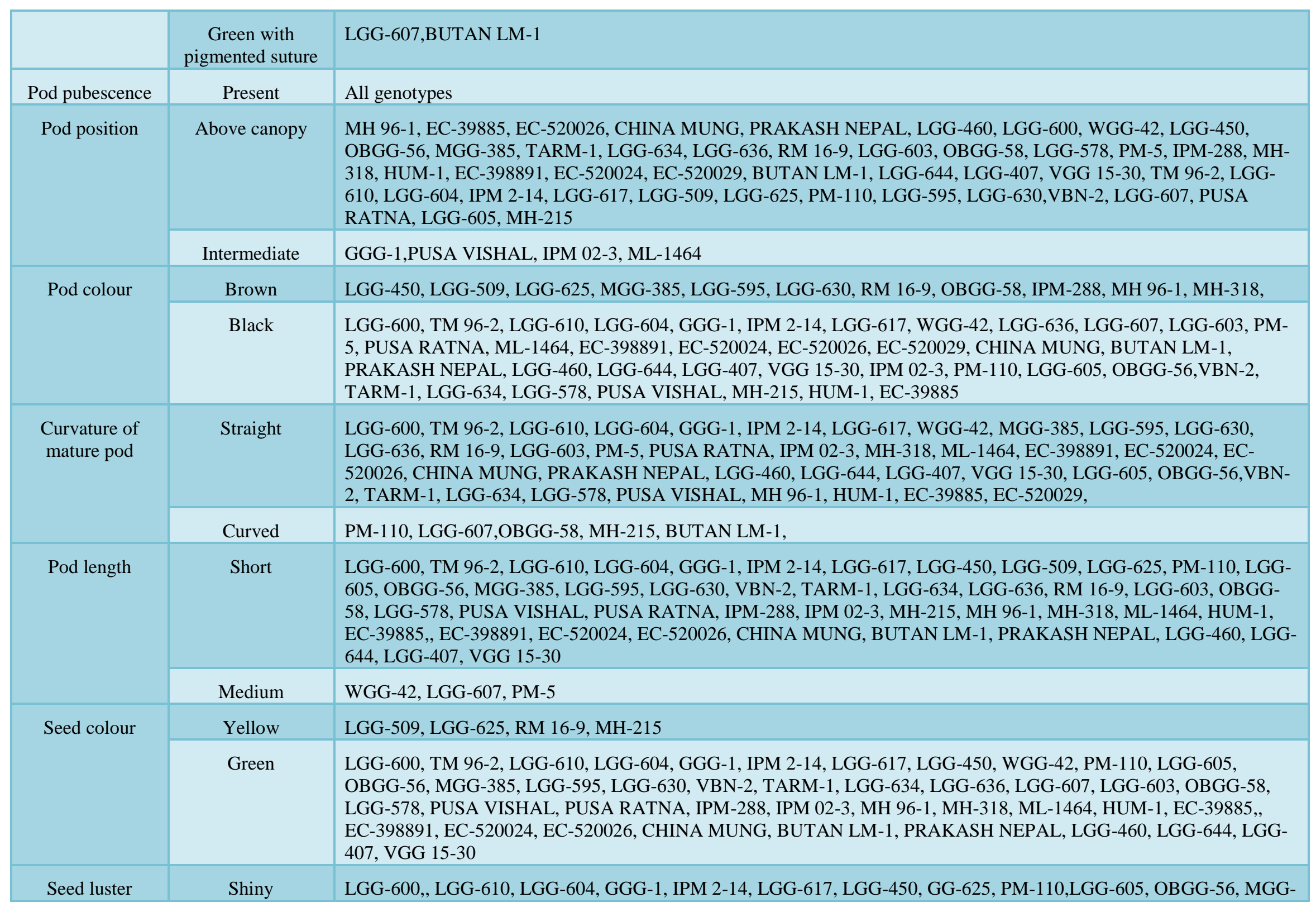




\begin{tabular}{|c|c|c|}
\hline & & $\begin{array}{l}\text { 385, LGG-595, LGG-630, VBN-2, TARM-1, LGG-634, LGG-607, RM 16-9, LGG-603, PM-5, PUSA VISHAL, IPM 02- } \\
\text { 3, MH-215, MH 96-1, HUM-1, EC-39885, EC-398891, EC-520024, EC-520026, EC-520029, CHINA MUNG, BUTAN } \\
\text { LM-1, PRAKASH NEPAL, LGG-460, LGG-644, VGG 15-30 }\end{array}$ \\
\hline & Dull & TM 96-2, WGG-42, LGG-509, LGG-636, OBGG-58, LGG-578, PUSA RATNA, IPM-288, MH-318, ML-1464, LGG-407 \\
\hline \multirow[t]{2}{*}{ Seed shape } & Oval & $\begin{array}{l}\text { TM 96-2, LGG-604, GGG-1, LGG-509, PM-110, OBGG-56, MGG-385, LGG-595, LGG-634, LGG-636, LGG-603, } \\
\text { OBGG-58, LGG-578, PUSA VISHAL, IPM-288, MH-318, LGG-460, LGG-407, VGG 15-30 }\end{array}$ \\
\hline & Drum & $\begin{array}{l}\text { LGG-600, LGG-610, IPM 2-14, LGG-617, LGG-450, WGG-42, LGG-625, LGG-605, LGG-630, VBN-2, TARM-1, } \\
\text { LGG-607, RM 16-9, PM-5, PUSA RATNA, IPM 02-3, MH-215, MH 96-1, ML-1464, HUM-1,, EC-39885, EC-398891, } \\
\text { EC-520024, EC-520026, EC-520029,, CHINA MUNG, BUTAN LM-1, PRAKASH NEPAL, LGG-644 }\end{array}$ \\
\hline \multirow[t]{3}{*}{ Seed size } & Small & GGG-1, LGG-450, TARM-1, LGG-636, RM 16-9, PUSA RATNA, MH-215, MH-318, LGG-460, LGG-644 \\
\hline & Medium & $\begin{array}{l}\text { LGG-600, TM 96-2, LGG-610, LGG-604, IPM 2-14, LGG-617, LGG-509, LGG-625, PM-110, LGG-605, OBGG-56, } \\
\text { MGG-385, LGG-595, LGG-630, VBN-2, LGG-634, LGG-607, LGG-603, OBGG-58, LGG-578, PUSA VISHAL, IPM- } \\
\text { 288, IPM 02-3, MH 96-1, ML-1464, HUM-1, EC-39885, EC-398891, EC-520024, EC-520026, EC-520029, CHINA } \\
\text { MUNG, BUTAN LM-1, PRAKASH NEPAL, LGG-407, VGG 15-30 }\end{array}$ \\
\hline & Large & WGG-42 \\
\hline
\end{tabular}


This is the trait which is highly used in the breeding programmes for differentiation of genotypes and also useful in maintenance breeding and Intellectual property protection. Similar exploitation of morphological traits in mungbean was reported by Mukherjee and Pradhan (2002), Khattak et al., (2000) and Patel et al., (2019).

The characters, time of flowering, plant habit and stem pubescence, were recorded at $50 \%$ flowering stage and variation among the genotypes was not observed. All the genotypes showed early flowering, determinate plant habit and presence of stem pubescence indicating they are not useful in characterization of these genotypes. The trait, growth habit, was semi erect type in most of the genotypes i.e., LGG-450, LGG-509, LGG-625, OBGG-56, TARM-1，LGG-634, LGG-636, LGG-607, RM 16-9, LGG-603, LGG-578, Pusa Ratna, IPM-288, IPM 02-3, IPM 02-14, MH 2-15, MH-318, ML-1464, HUM-1, EC-398885, EC-398891, EC520026, China Mung, LGG-460, LGG-644 and VGG 15-30 while, other genotypes exhibited erect growth habit. The character, stem colour was recorded at 50\% flowering stage and exhibited variation among the genotypes. The genotypes, LGG-600, TM 962, LGG-610, LGG-604, OBGG-56, MH 96-1, EC-520026 and VGG 15-30, stem colour was purple while the genotype, IPM-288, exhibited green with purple stem colour. The other genotypes recorded green stem colour.

Leaf lobes were present in the genotypes, LGG-600, VBN-2, RM 16-9 and VGG 15-30, while leaf lobes were absent in other genotypes. Leaf shape of the genotypes, LGG-600, VBN-, RM 16-9 and VGG 15-30, was cuneate while, other genotypes exhibited ovate shape. The genotypes, LGG-600, TM 96-2, LGG-610, LGG-604, IPM 2-14, LGG617, WGG-42, LGG-450, LGG-509, OBGG56, MGG-385, LGG-595, TARM-1, PM-5,
Pusa Vishal, MH 2-15 and HUM-1 showed dark green leaf colour while remaining genotypes recorded green colour. Leaf size of the genotypes, IPM 02-3, MH 2-15, MH 96-1, EC-398885, EC-520026, China Mung, Prakash Nepal and LGG-460, was small while, large leaf size was noticed in the genotypes, TM 96-2, LGG-610, LGG-604, GGG-1, IPM 2-14, LGG-617, LGG-509, LGG-625, PM-110， LGG-625， LGG-630, VBN-2, LGG-607 andvPusa Ratna. The remaining genotypes recorded medium leaf size.

The genotypes, TM 96-2, LGG-604, LGG617, PM-110, OBGG-56, LGG-630, VBN-2, LGG-634，LGG-636，LGG-607，LGG-578, IPM-288, IPM-288, IPM 02-14, MH 2-15, ML-1464, HUM-1, EC-398885, EC-520024, China Mung, Butan LM-1, showed purple coloured vein and greenish purple vein was observed in the genotypes, LGG-605 and EC398891. The remaining genotypes exhibited green vein colour. Petiole colour of the genotypes, LGG-610, GGG-1, LGG-450, LGG-509, LGG-625, OBGG-56, LGG-630, VBN-2, TARM-1， LGG-603， OBGG-58, PusaVisahl, Pusa Ratna, IPM 02-3, IPM 0214, MH 96-1, MH-318, ML-1464, EC520024, LGG-644, LGG-407 and VGG 1530, was greenish with purple colour, while LGG-605 exhibited purple petiole colour. Flower colour was light yellow in LGG-605 and $\mathrm{MH} 2-15$, while other genotypes showed yellow colour flower. Jain et al., (2002) reported the usefulness of flower characteristics in characterization of mungbean germplasm.

Premature pod colour was recorded when pods were fully developed and the colour was green with pigmented in Butan LM-1 while other genotypes recorded green colour of premature pod. Pod pubescence was present in all genotypes and there was no variation. Pod colour of the genotypes, LGG-450, LGG- 
625, MGG-385, LGG-630, RM 16-9, OBGG58, IPM-288,IPM 02-3, MH 96-1 and MH318 , was brown and the remaining genotypes showed black pod colour. Curvature of pod of the genotypes, PM-110, LGG-607, OBGG58, MH 2-15 and BUTAN LM-1, was curved while other genotypes exhibited straight pods. Similar report of straight pods without curvature was reported by Sunil et al., (2014) in their study in greengram. Pod position was intermediate in GGG-1, Pusa Vishal, IPM 023, IPM 02-14 and ML-1464, while other genotypes exhibited above canopy pod position. Pod length of WGG-42, LGG-607 and PM-5 genotypes was medium while, other genotypes exhibited short pod length.

Days to maturity was medium in LGG-610, LGG-607, RM 16-9, LGG-578, Pusa Vishal, Pusa Ratna, IPM 02-3, IPM 02 -14, MH 2-15, MH 96-1, EC-398885 and EC-398891 while, LGG-644 and LGG-407 recorded late maturity. Remaining genotypes showed early maturity. Plant height of the genotypes, TM 96-2, LGG-610, LGG-604, GGG-1, LGG450, LGG-509, LGG-625, PM-110, LGG605, MGG-385, LGG-630, LGG-636, LGG607, RM 16-9 and LGG-603, was medium and the other genotypes exhibited short plant height. Sunil et al., (2013) reported the variability in plant height in their study involving accessions from Andhra Pradesh.

The genotypes, LGG-509, LGG-625, RM 169 and $\mathrm{MH} 2-15$, recorded yellow seed colour while, other genotypes exhibited green seed colour. Seed luster of TM 96-2, WGG-42, LGG-509, LGG-636, OBGG-58, LGG-578, Pusa Ratna, IPM-288, IPM 02-14, MH-318, MH-1464 and LGG-407 was dull and the other genotypes exhibited shiny seed luster. Seed shape of the genotypes, TM 96-2, LGG604, GGG-1, LGG-509, PM-110, OBGG-56, MGG-385, LGG-595, LGG-634, LGG-636, LGG-603, OBGG-58, LGG-578, Pusa Vishal, IPM-288, MH-318, LGG-460, LGG-407 and
VGG 15-30, was oval while other genotypes exhibited drum seed shape. The trait, seed size (100 seed weight) was small in LGG450, TARM-1, LGG-636, RM 16-9, Pusa Vishal, IPM 02-14, MH 96-1, Prakash Nepal, LGG-460 and VGG 15-30 while WGG-42 genotype exhibited large seed shape. Remaining genotypes exhibited small seed shape (100 seed weight). Similar reports of exploiting the seed characters variability in mungbean was reported by Venkateswarlu (2001) and Khajudparn and Tantasawat (2011).

Thus, the present study indicated the importance of morphological characterization using DUS descriptors for the registration, maintenance and protection of genotypes. Anthocyanin pigmentation at seedling stage, stem, leaf, flower, pod and seed characters showed lot of variability among the genotypes.

\section{References}

Anumalla, M., Roychowdary, R., Geda, C., K and Mazid, M. 2015. Utilization of Plant Genetic Resources and Diversity Analysis Tools for Sustainable Crop Improvement with special emphasis on Rice International Journal of Advanced Research 3(3):1155-1175

Arunachalam, V. 1981. Genetic distance in plant breeding. Indian Journal of Genetics. 41: 226- 236

Indiastat, 2016-17.

Jain, S.K., D. Khare, M.S. Bhale and N.D. Raut. Characterization of mung bean varieties for verification of genetic purity. Seed Tech News. 32(1):200-201 (2002).

Kang, Y., T, Kim, S., K, Lee, S., H. 2014 Genome sequence of mungbean and insinght into evolution within Vigna species nature communication 5:1-9

Khajudparn, P. and P. Tantasawat: 
Relationships and variability of agronomic and physiological characters in mungbean. African $J$. Biotechnol., 10, 9992-10000 (2011).

Khattak, G.S.S., M.A. Haq, M. Ashraf and M. Saleem: Inheritance of hypocotyl colour and pubescence in mungbean (Vigna radiata (L.) Wilczek). J. Sci. Ind. Res., Iran, 11, 79-81 (2000).

Mukherjee, A. and K. Pradhan. Genetics of mungbean: I. Anthocyanin pigment in hypocotyl. J. Interacademicia., 6, 434437 (2002).

Patel, J. D., Patel, J.B. and Chetariya, C.P. 2019. Characterization of mungbean (Vigna radiata (L.) Wilczek) genotypes based on plant morphology. Indian J. Pure and App. Biosci., 7, 433-443 (2019).

Protection of Plant Varieties \& Farmers Rights Authority (2007).

Rao, V.R and Hodgkin, T. 2002. Genetic diversity and conservation and utilization of plant genetic resources. PCTOC. 68(1): 1-19.
Sunil, N., P. Someswara Rao, S. Natarajan, Jairam Reddy, S.K. Chakrabarty,J. Ashok and I.S. Bhist. Diversity in the landraces of greengram (Vigna radiata (L) R. Wilczek) collected from Tribal communities of Peninsular India. Photon. 114: 392-400 (2014).

Sunil, N., N. Sivaraj and S.K. Chakrabarty, Characterization and evaluation of mungbean (Vigna radiata L. Wilczek) germplasm from Andhr Pradesh. Indina J PGR 16: 18-20 (2013).

Venkateshwarlu, O.: Correlation and path analysis in greengram. Legume Res., 24, 115-117 (2001).

Wang, Y., Shahid, M.Q., Ghouri, F., Baloach, F.S., Wang, Y and Huang, H. 2015. Evaluation of the geographical pattern of genetic diversity of Glycine soja and Glycine max based on four single copy nuclear gene loci: For conservation of soybean germplasm. Biochemical Systematics and Ecology. 62: 229-235.

\section{How to cite this article:}

Mounika, S., J. V. Ramana, M. Lal Ahamed and Ratna Babu, D. 2020. Morphological Characterization of Greengram Germplasm using DUS Descriptors. Int.J.Curr.Microbiol.App.Sci. 9(09): 1701-1711. doi: https://doi.org/10.20546/ijcmas.2020.909.210 